\title{
Dependency of the "Shape" on Surface Morphology of Organized Molecular Films of Cyclic and Linear Block Copolymer of Polyethylene Oxide - Butyl Acrylate
}

\author{
Masamichi Hashimoto ${ }^{1}$, Qi Meng ${ }^{2}$, Satoshi Honda ${ }^{3}$, \\ Yasuyuki Tezuka ${ }^{4}$, Takuya Yamamoto ${ }^{4}$ and Atsuhiro Fujimori ${ }^{*}$ \\ ${ }^{1}$ Graduate School of Science and Engineering, and ${ }^{2}$ Department of Functional Materials Science, Saitama University \\ 255 Shimo-okubo, Sakura-ku, Saitama, 338-8570, Japan, \\ ${ }^{3}$ Faculty of Engineering, Tokyo University of Science \\ 1-2 Kagurazaka Shinjuku-ku Tokyo, 162-8601, Japan, \\ ${ }^{4}$ Graduate School of Science and Engineering, Tokyo Institute of Technology \\ 2-12-1 Ookayama, Meguro-ku, Tokyo, 152-8550, Japan. \\ * Corresponding author: Fax: +81-48-858-3503, e-mail: fujimori@fms.saitama-u.ac.jp
}

\begin{abstract}
A "topological polymer chemistry" at air/water interface is investigated by using an amphiphilic linear and cyclic block copolymers. A cyclic copolymer and two kind of linear polymers (AB-type diblock and ABA-type triblock copolymers) with same components were used in the study. The relatively stable monolayers of these three kinds of copolymers were formed at the air/water interface. The analogous condensed tendency and temperature dependency were observed in surface pressure-area isotherms of three kinds of monolayers. It is considered that molecular orientation at air/water interface of two kinds of liner block copolymers is similar to that of cyclic block copolymer. From the result of atomic force microscopic observation of transfered films, monolayers of the three kinds of polymers have formed a very similar morphology at mesoscopic scale under the conditions at room temperature and the constant compression speed. It finds that ABA type triblock liner copolymer formed fiber-like surface morphology by the two-dimensional crystallization based on the low compression speed. On the other hand, cyclic block copolymer formed shapeless domain.

Key words: Block copolymer, Cyclic polymer, Molecular orientation, Topological polymer chemistry, Langmuir-Blodgett film
\end{abstract}

\section{INTRODUCTION}

Presently, a hot attention is gathered with respect to changes in the properties of the polymer "shapes" as "linear", "branch", "cyclic", and "polycyclic" in the field of polymer science. ${ }^{1,2)}$ Therefore, when amphiphilic polymers with different "shape" have been spread to the air/water interface as monomolecular layer, effects on the formation and behavior of interfacial molecular films have researched in this study. ${ }^{3,4)}$ In the case of viewpoint of finding the merit of evaluation of this material properties, there is the fact that components of cell membranes of all including a human corresponds a linear lipid molecule. On the other hand, an archaea consists of cyclic polymer and has actually excellent heat resistance. ${ }^{5,6)}$

Meanwhile, in the category of traditional structure classification, this is the matter that cannot be understood. The "primary structure", "secondary structure", and "tertiary structure" is an established theory like as a "Don't teach your grandmother to suck eggs" to the polymer science-specialized researchers in the concept of the corresponding field. ${ }^{7,8)}$ The "primary structure" corresponds chemical structure including molecular weight. "Secondary structure" correspond conformation by intermolecular interaction such as cis-trans, trans-gauche, and helical conformation. And a "tertiary structure" correspond crystal, amorphous and liquid crystal, etc. "Higher-order structure" correspond the matter of fusion of secondary and tertiary structures, such as spherulites and phase separation.

Three types of amphiphilic block copolymer of butyl acrylate / polyethylene oxide have used in this study. These materials have a difference of ring-shaped or two-type linear block copolymers composed by same monomer species. Molecular arrangement and packing of such like polymer species at air/water interface and on solid substrate are very interesting to basic academic filed. In this study, a "topological polymer chemistry" at air/water interface is investigated by using an amphiphilic linear (AB-type diblock and ABA-type triblock) and cyclic block copolymers.

\section{EXPERIMENTAL}

\subsection{Materials}




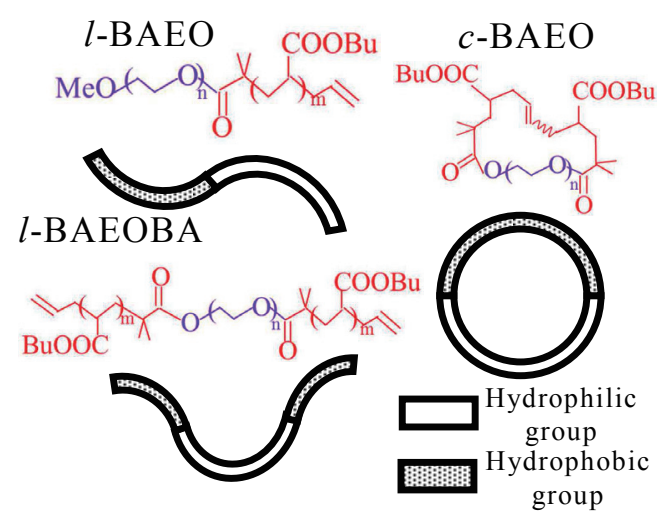

Figure 1 Chemical structures of $l$-BAEO $l$-BAEOBA and $c$-BAEO.

Figure 1 schematically shows the detailed information of compounds used in this study. The cyclic copolymer is abbreviated by $c$-BAEO. The $c$-BAEO, AB-type (l-BAEO), and ABA-type ( $l$-BAEOBA) block copolymers are composed by butyl acrylate and polyethylene. Chemical structure of cyclic polymers correspond the connecting shape of both ends of the ABA type. The molecular weights of all polymers indicate less than 10,000, and these polymers are able to be solved in the chloroform solvent. In order to form the monolayer on the water surface, these polymers have spread on the ultrapure water surface from their chloroform solution.

\subsection{Measurement}

Surface pressure - area $(\pi-A)$ isotherms were measured by using a Langmuir trough (USI, USI-3-22) in order to evaluate the monolayer behaviors on the water surface. Further, in order to observe the surface morphology of the transferred monolayer on solid substrate, atomic force microscopy (AFM) observation was performed.

\section{RESULTS AND DISCUSSION}

Figure 2 has shown $\pi$-A isotherms related to the monolayer behavior of the three kind of shapes of block copolymers. The mean area per monomer units as horizontal axis has been expressed. The temperature of subphase is $15{ }^{\circ} \mathrm{C}$. At a first glance, all three polymers commonly show the analogical isotherms with about $20 \AA^{2}$ the limiting area of condensed phase and below $30 \mathrm{mNm}^{-1}$ collapsed surface pressure. Since the values of horizontal axis are considerably smaller as the limiting area per monomer, it seems an unnatural value. (The values of horizontal axis have to consider as twice value because two hydrophobic polymer chains is across the air/water interface, like a insert illustration in Fig. 2). The reason of drawn the analogical illustrations of three polymers indicates no choice to some extent of conformation of polymers at air/water interface under the similar results of individual isotherms. In other words, it is presumed that two-kind of
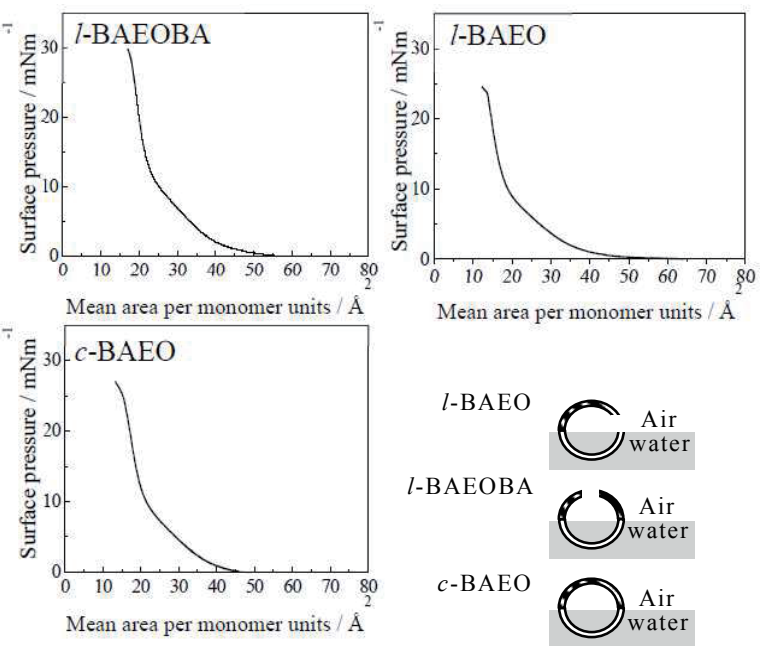

Figure $2 \pi$-A isotherms of monolayer on the water surface for several copolymers $\left(15^{\circ} \mathrm{C}\right)$.

liner polymers form the similar conformation to the cyclic polymer at interface. In this stage, It is considered that the expansion behavior of the linear polymers which the terminal is not connected, is more remarkable than that of the cyclic polymers. In the Fig. 3, results of this measurement with a rising of subphase temperature are shown. This figure shows the change in the isotherms at subphase temperature of 15,25 , and $35^{\circ} \mathrm{C}$. All isotherms indicate an expansion tendency together with the increasing of subphase temperature. However, since only this figure is hard to understand of the differences of characteristics of each polymer, actual values summarize in the table 1 . Table 1 summarizes the limiting area of condensed phase and the expansion phase with the collapse surface pressure. This data quantitatively indicates that three kinds of polymers also have taken a similar behavior and surface conformation of linear polymers does not raise a significant disturbance till the $35{ }^{\circ} \mathrm{C}$. As a surprising matter, amphiphilic polymers with different shapes indicate high similarity of monolayer behavior
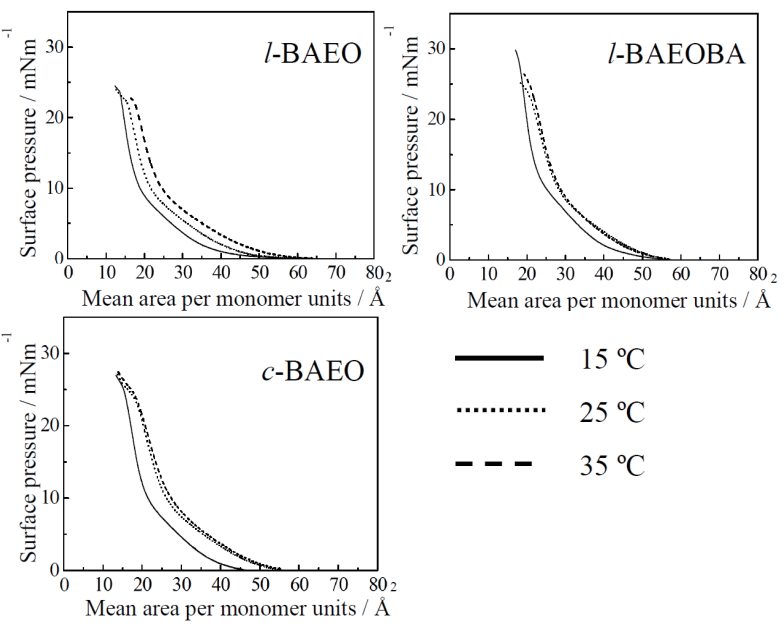

Figure $3 \pi$-A isotherms of monolayer on the water surface for several copolymers $\left(15-35^{\circ} \mathrm{C}\right)$. 
Table 1 Limiting areas and collapsed pressures for several copolymers.

$l$-BAEO
\begin{tabular}{|c|c|c|c|}
\hline & $\begin{array}{c}\text { Primary } \\
\text { condensed phase } \\
\text { limiting area }\left(\AA^{2}\right)\end{array}$ & $\begin{array}{c}\text { Secondary } \\
\text { condensed phase } \\
\text { limiting area }\left(\AA^{2}\right)\end{array}$ & $\begin{array}{c}\text { Collapsed } \\
\text { pressure } \\
\left(\mathrm{mNm}^{-1}\right)\end{array}$ \\
\hline $15{ }^{\circ} \mathrm{C}$ & $\mathbf{3 8}$ & $\mathbf{2 4}$ & $\mathbf{2 3}$ \\
\hline $25{ }^{\circ} \mathrm{C}$ & $\mathbf{4 2}$ & $\mathbf{3 0}$ & $\mathbf{2 2}$ \\
\hline $35^{\circ} \mathrm{C}$ & $\mathbf{4 5}$ & $\mathbf{3 1}$ & $\mathbf{2 2}$ \\
\hline
\end{tabular}

l-BAEOBA
\begin{tabular}{|c|c|c|c|}
\hline & $\begin{array}{c}\text { Primary } \\
\text { condensed phase } \\
\text { limiting area }\left(\AA^{2}\right)\end{array}$ & $\begin{array}{c}\text { Secondary } \\
\text { condensed phase } \\
\text { limiting area }\left(\AA^{2}\right)\end{array}$ & $\begin{array}{c}\text { Collapsed } \\
\text { pressure } \\
\left(\mathrm{mNm}^{-1}\right)\end{array}$ \\
\hline $15{ }^{\circ} \mathrm{C}$ & $\mathbf{3 8}$ & $\mathbf{2 4}$ & $\mathbf{2 3}$ \\
\hline $25{ }^{\circ} \mathrm{C}$ & $\mathbf{4 2}$ & $\mathbf{3 0}$ & $\mathbf{2 2}$ \\
\hline $35^{\circ} \mathrm{C}$ & $\mathbf{4 5}$ & $\mathbf{3 1}$ & $\mathbf{2 2}$ \\
\hline
\end{tabular}

\begin{tabular}{|c|c|c|c|}
\multicolumn{1}{c|}{$c$-BAEO } & $\begin{array}{c}\text { Primary } \\
\text { condensed phase } \\
\text { limiting area }\left(\AA^{2}\right)\end{array}$ & $\begin{array}{c}\text { Secondary } \\
\text { condensed phase } \\
\text { limiting area }\left(\AA^{2}\right)\end{array}$ & $\begin{array}{c}\text { Collapsed } \\
\text { pressure } \\
\left(\mathrm{mNm}^{-1}\right)\end{array}$ \\
\hline $15{ }^{\circ} \mathrm{C}$ & $\mathbf{3 8}$ & $\mathbf{2 4}$ & $\mathbf{2 3}$ \\
\hline $25^{\circ} \mathrm{C}$ & $\mathbf{4 2}$ & $\mathbf{3 0}$ & $\mathbf{2 2}$ \\
\hline $35^{\circ} \mathrm{C}$ & $\mathbf{4 5}$ & $\mathbf{3 1}$ & $\mathbf{2 2}$ \\
\hline
\end{tabular}

and dependency of subphase temperature even if the components of polymers are same.

The next three figures show the morphological changes at mesoscopic scale in the monolayers on solid substrate by upstroke of LB method. All figures have measured at $5 \mu \mathrm{m} \times 5 \mu \mathrm{m}$ scales. Figure 4 shows AFM images of Z-type monolayers of three types block copolymers. These monolayers have been observed in the liquid condensed phase at low surface pressure region. The morphology of monolayer of $l$-BAEO indicates domain structure. l-BAEOBA and $c$-BAEO show the bend line-shaped structure at $15{ }^{\circ} \mathrm{C}$ subphase temperature. It looks that aggregation tendency of $\mathrm{AB}$ type diblock copolymers are relatively remarkable at first glance, however, significant difference has not been observed in the formation process of all monolayers in these mesoscopic scales.

As the next attempt, morphological observation under the increasing of subphase temperature was performed. Figure 5 shows AFM images of Z-type monolayers of three types block copolymers at $35{ }^{\circ} \mathrm{C}$. The changes in aggregation tendency as mentioned above have also noticed here. Although $\pi$-A isotherms could not confirm at the scale of the that measurement, the aggregation tendency of cyclic polymer has the highest than others in monolayer, and ABA type triblock linear polymer remains

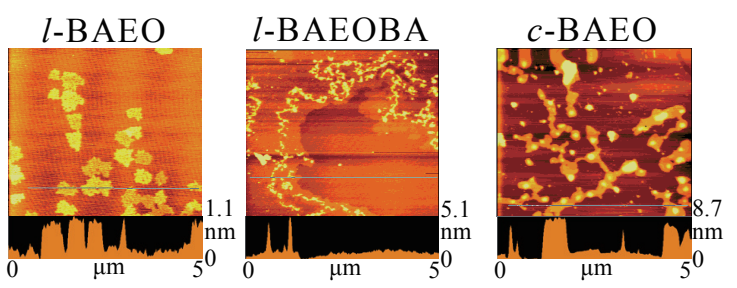

Figure 4 AFM images of Z-type monolayers for several copolymers $\left(15^{\circ} \mathrm{C}, 15 \mathrm{mNm}^{-1}, 10 \mathrm{~cm}^{2} / \mathrm{min}\right)$.
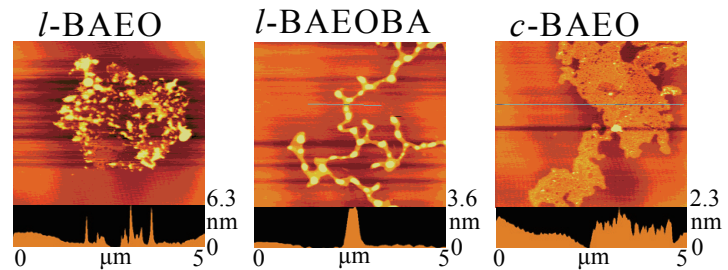

Figure 5 AFM images of Z-type monolayers for several copolymers $\left(35^{\circ} \mathrm{C}, 15 \mathrm{mNm}^{-1}, 10 \mathrm{~cm}^{2} / \mathrm{min}\right)$.

still a network forms. When attention is paid to these "difference between the aggregation trends", the experiment with an accelerating of the spontaneous morphogenesis on the water surface has performed the reducing of the compression speed to the monolayer till $1 / 10$. Figure 6 shows AFM images of Z-type monolayers of three types block amphiphilc copolymers at slow compression speed. In this case, the obvious difference is confirmed. The monolayer of ABA type triblock copolymer spontaneously and slowly grows as a fibrous domain at the air/water surface and forms a nano-fiber. In the AB type diblock copolymer as liner copolymer, spontaneous structural growth does not been confirmed and their film quality is rather reduced, only. Compared to these liner copolymers, the monolayer of cyclic polymer has begun to form the state as the precursor which domain connects each other after formation of domain as individual nuclear. Since two terminal hydrophobic chains of ABA type triblock copolymer have oriented to the air, it is suggested that two-dimensional developed characteristic morphology is easy to be produced by inter-terminal chains interaction (Fig. 7).

It is important point in this study that two kind of linear copolymers orient a same configuration of cyclic polymers at the air/water interface. However, from the influence on the induction of spontaneous structural formation based on the dependency of compression rate at the air/water interface, nano-fiber formation is shown in ABA triblock linear copolymer, which this phenomenon cannot be confirmed in another polymer films. In this study, although there is a lot of content of preliminary experiment, it finds that dependency of "shape" of polymers as shown in archaea and a lipid in the other organism become a factor influenced to the forms and functionality in the two-dimensional organization.

Indeed, in the case of multilayer formation by
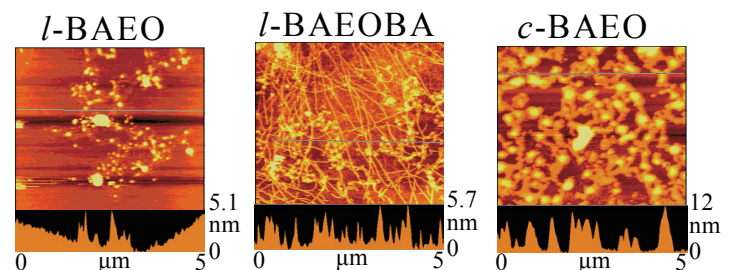

Figure 6 AFM images of Z-type monolayers for several copolymers $\left(15^{\circ} \mathrm{C}, 15 \mathrm{mNm}^{-1}, 1 \mathrm{~cm}^{2} / \mathrm{min}\right)$. 


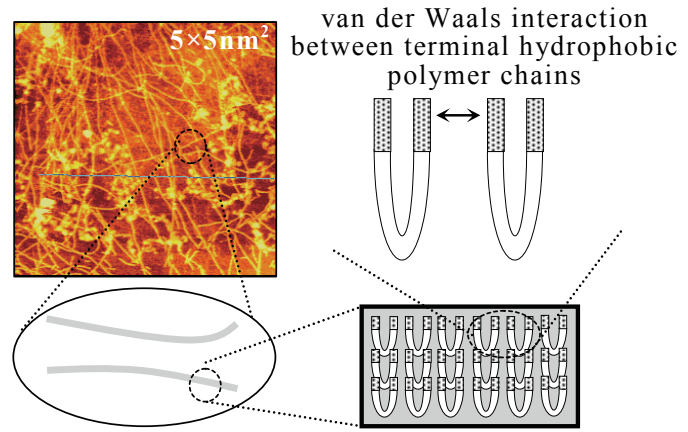

Figure 7 Schematic illustration of formation of fiber like structure.

accumulation of these monolayers, it is easy to form resemble periodic structure based on their interfacial configuration. In addition, in the estimation of heat-resistant of multilayers, experimental fact is obtained that layer structure of a cyclic polymer is extremely stable to the heat. 9) These experimental results cede to the other articles in the same issue of publication of this journal. ${ }^{10)}$

\section{CONCLUSION}

A topological effect of polymers at the air/water interface is investigated by using an amphiphilic linear and cyclic block copolymers. A cyclic copolymer, AB-type diblock linear copolymer, and ABA-type triblock copolymers with same components were newly synthesized and used in the study. All the copolymers form the relatively stable monolayers at the air/water interface. The similar condensed tendency and temperature dependency were observed in $\pi$-A isotherms of three kind of monolayers. It is considered that molecular orientation at air/water interface of two kinds of liner block copolymers is same to that of cyclic block copolymer. From the result of AFM observation of monolayers on solid, monomolecular films of the three kind of copolymers have formed a resemble morphology at mesoscopic scale under the conditions at room temperature and the constant compression speed. It finds that $\mathrm{ABA}$ type triblock liner copolymer formed fibril structure by the two-dimensional crystallization based on the low compression speed. A cyclic block copolymer formed aggregated domain.

\section{REFERENCES}

[1] S. Honda, T. Yamamoto, Y. Tezuka, J. Am. Chem. Soc., 132, 10251, (2010).

[2] S. Honda, T. Yamamoto, Y. Tezuka, Nature. Commun., 4, 1574, (2013).

[3] K. Adachi, S. Honda, S. Hayashi, Y. Tezuka, Macromolecules, 41, 7898, (2008).

[4] E. Baba, S. Honda, T. Yamamoto, Y. Tezuka, Polym. Chem., 3, 1903, (2012).

[5] K. Arakawa, T. Eguchi, K. Kakinuma, Bull. Chem. Soc. Jpn., 74, 347, (2001).

[6] A. Yamagishi, Biol. Sci. Space, 14, 332, (2000).

[7] GR. Strobl, M. Schneider, J. Polym. Sci. B, Polym. Phys., 18, 1343, 1980.
[8] A. Keller, Philos. Mag., 2, 1171, (1957).

[9] A. Fujimori, S. Arai, Y. Soutome, M. Hashimoto, Colloids Surf. A, (2014), in press.

[10] Q. Meng, M. Hashimoto, S. Honda, Y. Tezuka, T. Yamamoto, A. Fujimori, Trans. Mater. Res. Soc. Jpn., 39, (2014), to be submitted.

(Received February 7, 2014; Accepted February 19, 2014) 